\title{
Specialty-Related Disparities of Readmission in Patients with Chronic Heart Failure: The Importance of Hospital-Clinic Cooperation
}

\author{
Masayoshi Sakakibara, Ken Kongoj, Hisanori Samejima, Kunio Shiota, Akihiko Takagi, \\ Fumihiko Miyake and Masahiro Murayama
}

\begin{abstract}
Object The purpose of this study was to elucidate differences in readmission rates and late outcome in outpatients with chronic heart failure treated in different clinical settings. Patients and Methods This study included 65 consecutive patients who were admitted to our CCU due to acute heart failure for the first time and discharged from our institution. After their discharge, 31 were cared for by a cardiologist in the outpatient clinic of our institution (group A) and the other 34 were cared for by a general practitioner in a clinic (group B). The various findings during the acute phase and the follow-up period were retrospectively compared between the two groups. In addition, the incidence of unexpected readmission and prolonged outcomes were compared between the two groups. Results The patients in group B were older than those in group A, but no other differences were noted in patient characteristics. More patients in group A required more than one hospitalization within 6 months from discharge (group A, 35.5\%; group B, 8.9\%, p<0.01; follow-up period, 17.1 \pm 5.9 months). There was no difference in the survival rate between the groups. Conclusion We concluded that stabilized outpatients should receive comprehensive care from a general practitioner to avoid the need for readmission after discharge.
\end{abstract}

(Internal Medicine 38: 705-709, 1999)

Key words: comprehensive management, late outcome, prevention of readmission

\section{Introduction}

Congestive heart failure is a major public health problem that affects more than 2 million people and causes 200,000 deaths annually in the United States. It was reported that hospital admissions in the United State have reached 1 million each year, incurring annual medical costs in excess of an estimated $\$ 7$ billion (1). Therefore, prevention of readmissions for heart failure is an extremely important task. Contrary to the case in the United States, there are no detailed reports on hospital admissions for congestive heart failure in Japan. However, according to the medical certificates of death in the 1996 vital statistics of Japan, heart failure caused approximately 40,000 deaths in that year (2). On the assumption that $20 \%$ of all patients with heart failure die, 200,000 patients in Japan suffer from heart failure each year. This incidence suggests that the problem is almost as serious in Japan as it is in the United States. Patients with severe heart disease require intensive lifesaving care as soon as possible in the acute phase. In contrast, avoidance from precipitating factors is required for outpatients with chronic heart failure. Therefore, management by a cardiologist in an advanced institution and comprehensive care by a general practitioner in a clinic are both important for stable outpatient care. Accordingly, cooperation between the general practitioner and cardiologist is imperative. Nonetheless, there have been few reports on the management of outpatients with chronic heart failure.

This study was conducted to elucidate the differences in readmission rates and late outcome in patients with stable chronic heart failure when treated by a general practitioner in a clinic or a cardiologist in a cardiological institution.

\section{Subjects and Methods}

This study included 65 consecutive patients who were admitted to our CCU due to acute heart failure for the first time and discharged from our institution between January 1, 1996 and March 30, 1997. After discharge, 31 were cared for by a

From the Second Department of Internal Medicine, St. Marianna University of Medicine, Kanagawa

Received for publication October 13, 1998; Accepted for publication May 2, 1999

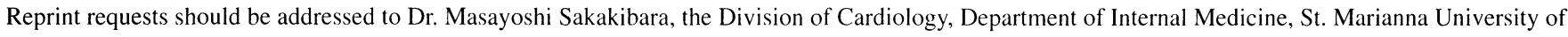
Medicine, 2-16-1 Sugao, Miyamaeku, Kawasaki 216-0015 
cardiologist in our institution (group A) and the remaining 34 were cared for by a general practitioner in a clinic (group B). The following items were retrospectively compared between the two groups: 1) physical findings, 2) hemodynamics, 3) use of artificial respirator on admission as a parameter of disease severity, 4) length of stay in CCU, 5) left ventricular ejection fraction, 6) pharmacotherapies at the period of discharge, 7) incidence of unexpected readmission, 8) compliance with medications and dietary restrictions among the patients requiring readmission. In addition, the late outcomes were compared between 28 patients from group A and 17 from group B who could still be followed in March 1998. The data on group B was acquired from the general practitioners treating the patients.

Values are expressed as the mean $\pm \mathrm{SD}$. Continuous variables were analyzed by the Student's t test. The chi-square test or Mann-Whitney's U test was used to analyze categoric variables between the groups. Survival ratios were calculated using the Kaplan-Meier product limit. The log-rank test was applied for evaluating differences in the survival ratio between the groups. For all comparisons, $\mathrm{p}<0.05$ was considered significant.

\section{Results}

The clinical characteristics of the 2 groups are summarized in Table 1. There were no significant differences in gender between the groups, but the patients in group B were significantly older than those in group A (group A, 67.0 13.6 years; group B, 78.6 \pm 9.8 years, $p<0.01$ ). The etiology of heart failure did not differ significantly between the groups. About half of the patients in each group had complex causes such as hypertensive heart disease or respiratory infection (group A, 45.2\%; group B, 41.2\%). Figure 1A shows the index of severity on admission in accordance with the Killip classification (3). In

Table 1. Patient Characteristics

\begin{tabular}{ccccc}
\hline & Total & Group A & Group B & \\
\hline $\mathrm{n}$ & 65 & 31 & 34 & \\
Gender $(\mathrm{m} / \mathrm{f})$ & $37 / 28$ & $19 / 12$ & $18 / 16$ & $\mathrm{~ns}$ \\
Age $(\mathrm{yr})$ & $73.0 \pm 13.2$ & $67.0 \pm 13.6$ & $78.6 \pm 9.8$ & $\mathrm{p}<0.01$ \\
Etiology & 12 & 5 & & \\
Coronary artery disease & 13 & 6 & 7 & \\
Valvular heart disease & 8 & 4 & 4 & \\
Myopathy* & 2 & 1 & 1 & $\mathrm{~ns}$ \\
Rapid atrial fibrillation & 28 & 14 & 14 & \\
Complex & 2 & 1 & 1 & \\
Others & & &
\end{tabular}

*include hypertrophic cardiomyopathy, dilated cardiomyopathy and hypertensive heart disease.
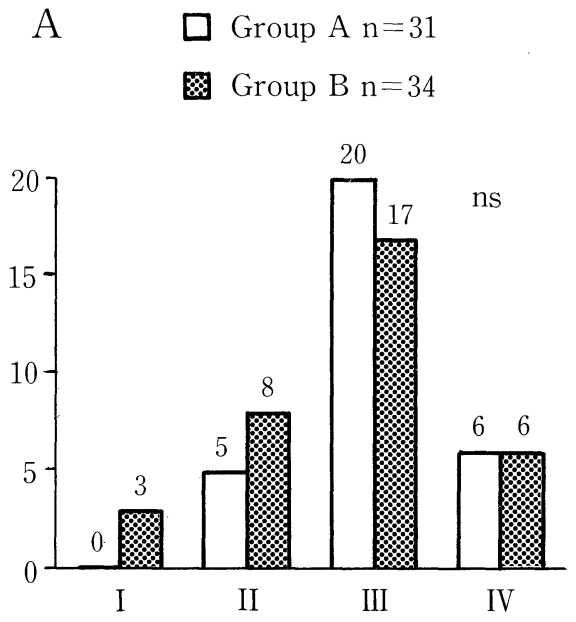

B $\square$ Group $\mathrm{A} \mathrm{n}=17$

Group B n=14

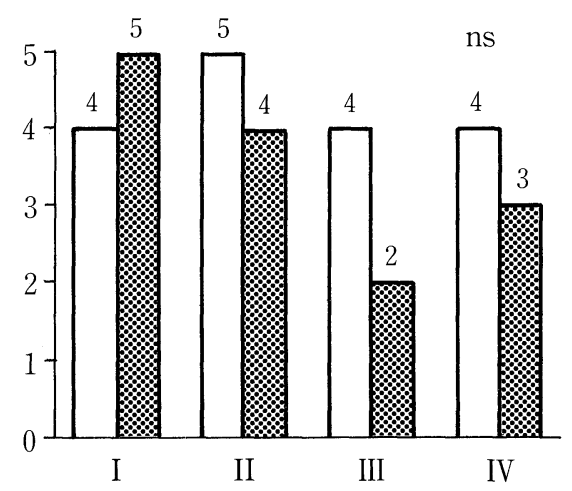

Figure 1. A: The index of severity on admission according to the Killip classification. No significant difference was noted between the groups.B: The index of hemodynamic severity on admission according to the Forrester subset. There was no difference in hemodynamics between the groups. 


\section{Readmission of Chronic CHF}

group A, $0(0 \%), 5(16.1 \%), 20(64.5 \%)$, and $6(19.4 \%)$ patients were class I, II, III, and IV, respectively. In group B, 3 $(8.8 \%), 8(23.5 \%), 17(50.0 \%)$, and $6(17.6 \%)$ patients were class I, II, III, and IV, respectively. No significant differences were noted between the groups. A Swan-Ganz catheter had been required in 17 cases $(54.8 \%)$ in group $A$ and in 14 cases $(41.2 \%)$ in group B. The index of hemodynamic severity on admission in accordance with the Forrester subset (4) is shown in Fig. 1B. In group A, patients of subset I, II, III, and IV were $4(23.5 \%), 5(29.4 \%), 4(23.5 \%)$, and $4(23.5 \%)$, respectively; in group B, patients of subset I, II, III, IV were $5(35.7 \%), 4$ $(28.6 \%), 2(14.3 \%)$, and $3(21.4 \%)$, respectively. There was no difference in Forrester subset between the groups. A respirator had been used at the acute phase in 5 patients $(16.1 \%)$ in group $\mathrm{A}$, and in 4 patients $(11.8 \%)$ in group $\mathrm{B}$; no significant difference in the requirement for a respirator was noted. There was no difference in the length of stay in CCU between the groups (group A, 5.7 \pm 4.0 days; group B, $4.4 \pm 3.3$ days). The left ventricular ejection fraction assessed by 2 -dimensional echocardiography at the predischarge period was similar in both

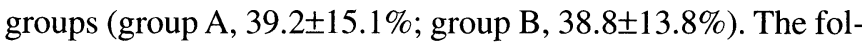
lowing pharmacotherapies at the discharge were compared between the groups: diuretics, angiotensin-converting enzyme inhibitors and positive inotropic agents other than digitalis glycosides (Fig. 2). The utilization rates of diuretics, angiotensinconverting enzyme inhibitors and positive inotropic agents were $74.2 \%, 67.7 \%$, and $6.5 \%$, respectively, in group A and $88.2 \%$, $64.7 \%$, and $2.9 \%$, respectively, in group B, with no significant difference between the groups. The difference in unexpected readmission between the groups is shown in Fig. 3. The observed period was $17.1 \pm 5.9$ months and did not differ significantly between the groups (group A, 18.3 \pm 5.2 months; group $\mathrm{B}, 15.2 \pm 6.1$ months). There were fewer patients not requiring readmission in group A than in group B (group A: $51.6 \%$ vs.

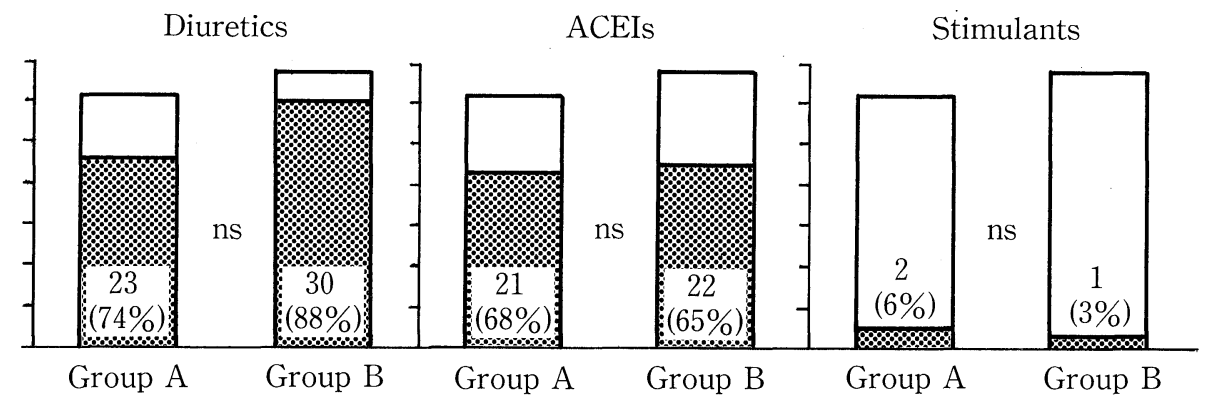

Figure 2. Pharmacotherapies at discharge. There was no significant difference between the groups. ACEIs: Angiotensin-converting enzyme inhibitors, Stimulants: positive inotropic agents.

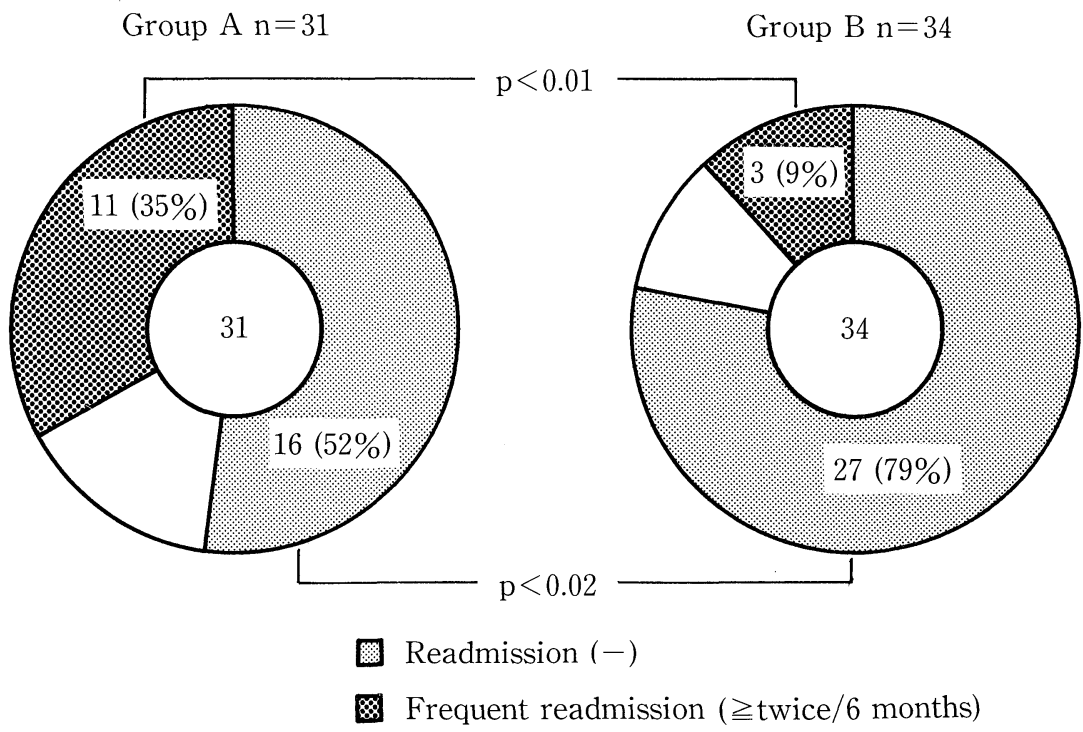

Figure 3. Difference of unexpected readmission between the groups. Patients not requiring rehospitalization were significantly fewer in group $A$ than in group B. Significantly more patients in group A required more than one rehospitalization within 6 months. 
Table 2. Compliance of Medication and Diet

\begin{tabular}{cccc}
\hline & Group A & Group B & \\
\hline Good & 6 & 4 & \\
Common & 4 & 1 & \\
Low & 5 & 2 & ns \\
\hline
\end{tabular}

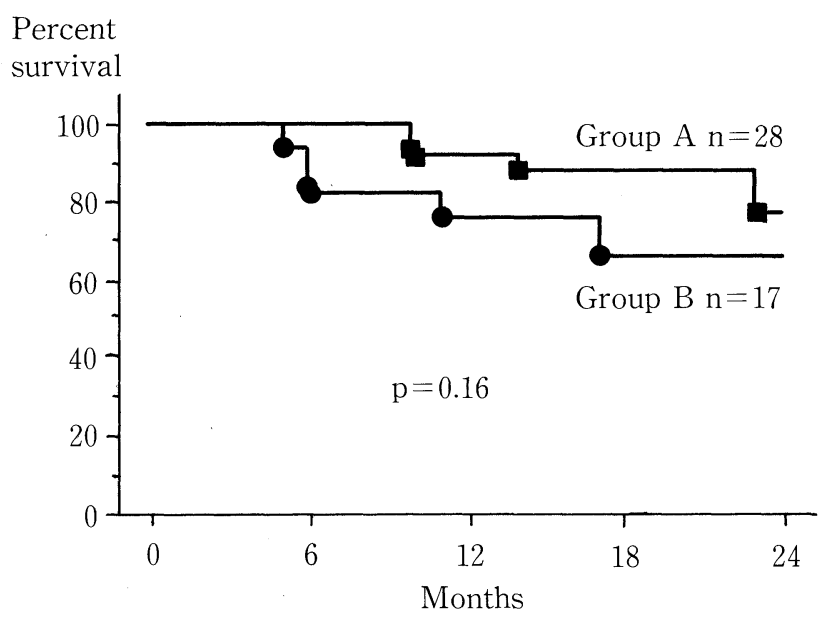

Figure 4. Comparison of survival rate within 24 months after the discharge between the groups. Four patients in group $\mathbf{A}$ and 5 in group $B$ died, but no significant difference was noted between the groups.

group B: $79.4 \%, \mathrm{p}<0.02$ ). In addition, there were more patients in group $\mathrm{A}$ who required more than one rehospitalization within 6 months (group A, 35.5\%; group B, 8.9\%, p $<0.01$ ). Compliance with medications and dietary restrictions among the patients requiring readmission is shown in Table 2. There was no significant difference between the groups. The survival rate within 24 months after the initial discharge is shown in Fig. 4. Four patients in group A and 5 in group B died, but no significant difference was noted between the groups.

\section{Discussion}

Urgent intensive care in the core institution is required for patients with severely acute heart disease (5-7). However, an earlier study (8) reported that among cases requiring rehospitalization, the most frequent reason for the rehospitalization was noncompliance with medications and dietary restrictions. Also, Monane et al reported that only $10 \%$ of their study population filled enough prescriptions to have daily medication available for the entire year of follow-up (9). Therefore, avoidance from the precipitating factors is very important for patients after their therapies have been completed and their therapeutic plans have been determined. Both management by a cardiologist in an advanced institution in the acute phase and comprehensive care for the stable outpatient by a general practitioner in a clinic are important. The comprehensive care is especially important in elderly cases (10).

Since the patients included in our study entered the CCU of an academic medical institution with an emergency center, all of them received intensive care and diagnostic examinations immediately upon admission. Among the outpatients treated after discharge, some patients were cared for by cardiologists in our hospital and others were cared for by practitioners at nearby clinics at the patient's or family's request. Since the residences and the activities of the patients may have affected this selection, these patients were not randomly assigned to groups A and B. However, in spite of the difference in age there were no significant differences in patient characteristics, severity of disease, length of stay in CCU, cardiac function, and pharmacotherapies. In addition, summaries with detailed information on the basal heart disease, therapies, and future care points were supplied to the general practitioners at the time of discharge. Therefore, we believe that there was no fundamental difference in contents of therapies between the two groups after discharge. Previous studies (11-13) reported that an intensive home-care program was associated with a marked decrease in the need for hospitalization and an improved functional status of patients with heart failure. The present study indicated that more patients required frequent rehospitalization in group A than in group B. This finding may be a reflection of the strict care practiced by general practitioners, for example, frequent examinations or visits to the patients. There are many reports $(5,6)$ on specialty-related disparities in the managements of heart diseases. Reis et al (7) demonstrated that overall mortality at 6 months after discharge did not differ between the patients cared for by a generalist and those cared for by a cardiologist, but that the former were more likely to be readmitted. Their report yielded a different result from the present study. However, their previous study was a comparison between initial managements by a non-specialist and a cardiologist. In contrast, our study was a comparison of the care for outpatients with stabilized heart failure after a specialist had performed the initial management, diagnostic examinations, and, when necessary, invasive procedures.

We concluded that hospitalized patients with acute heart failure should be treated by a cardiologist, while outpatients with stabilized heart failure, especially those advanced in years, should be cared for by a general practitioner to prevent readmission.

\section{Study Limitations}

The present study is a retrospective investigation with a small limited sample size. The patients were not randomly assigned to groups $\mathrm{A}$ and $\mathrm{B}$, and the data on the late outcome was incomplete. Although a large scale randomized study is needed to determine the appropriate roles for cardiologists and general practitioners, this study did indicate the actual state of treatment of heart failure in Japan and the future problem of different roles for cardiologists and general practitioners in the management of outpatients with chronic heart failure. 


\section{Readmission of Chronic CHF}

\section{References}

1) Konstam M, Dracup K, Baker D, et al. Heart failure: Evaluation and care of patients with left-ventricular systolic dysfunction. Clinical practice guideline No. 11. AHCPR Publication No. 94-0612. Agency for Health Care Policy and Research, Public Health Service, Department of Health and Human Services. Rockville, MD, 1994: 1-9.

2) Statistics and Information Department of the Minister's Secretariat. Vital Statistics of Japan. Statistics and Information Department of the Minister's Secretariat in the Ministry of Health and Welfare, Tokyo, 1998: 172-175 (in Japanese).

3) Killip T, Kimball JT. Treatment of myocardial infarction in a coronary care unit. Am J Cardiol 20: 457-464, 1967.

4) Forrester JS, Diamond GA, Swan HJC. Correlative classification of clinical and hemodynamic function after acute myocardial infarction. Am J Cardiol 39: 137-145, 1977.

5) Ayanian JZ, Hauptman PJ, Guadagnoli E, Antman EM, Pashos CL, McNeil BJ. Knowledge and practices of generalist and specialist physicians regarding drug therapy for acute myocardial infarction. N Engl J Med 331: 1136-1142, 1994.

6) Schreiber TL, Elkhatib A, Grines CL, O'Neill WW. Cardiologist versus internist management of patients with unstable angina: treatment patterns and outcome. J Am Coll Cardiol 26: 577-582, 1995.
7) Reis SE, Holubkov R, Edmundowicz D, et al. Treatment of patients admitted to the hospital with congestive heart failure: specialty-related disparities in practice patterns and outcome. J Am Coll Cardiol 30: 733738, 1997.

8) Ghali JK, Kadakia S, Cooper R, Ferlinz J. Precipitating factors leading to decompensation of heart failure. Traits among urban blacks. Arch Inter Med 148: 2013-2016, 1988.

9) Monane M, Bohn RL, Gurwitz JH, Glynn RJ, Avorn J. Noncompliance with congestive heart failure therapy in the elderly. Arch Intern Med 154: 433-437, 1994.

10) Vinson JM, Rich MW, Sperry JC, Shah AS, McNamara T. Early readmission of elderly patients with congestive heart failure. J Am Geriatr Soc 38: 1290-1295, 1990.

11) Cintron G, Bigas C, Linares E, Aranda JM, Hernandez E. Nurse practitioner role in a chronic congestive heart failure clinic: In-hospital time, cost, and patient satisfaction. Heart Lung 12: 237-240, 1983.

12) Kornowski R, Zeeli D, Averbuch M, et al. Intensive home-care surveillance prevents hospitalization and improves morbidity rates among elderly patients with severe congestive heart failure. Am Heart J 129: 762766, 1995.

13) Fonarow GC, Stevenson LW, Walden JA, et al. Impact of a comprehensive heart failure management program on hospital readmission and functional status of patients with advanced heart failure. J Am Coll Cardiol 30: 725-732, 1997. 